\title{
EXPERIMENTAL AND NUMERICAL STUDIES OF A DIRECT CURRENT MICRODISCHARGE PLASMA THRUSTER
}

\section{U. KC, T. Deconinck, P. L. Varghese, and L. L. Raja}

ASE-EM Department

The University of Texas at Austin Austin, Texas 78712, USA

\begin{abstract}
A simple Microdischarge Plasma Thruster (MPT) for small satellite propulsion has been designed and studied by performing experiments and running simulations with a numerical model. The MPT comprises a tri-layer sandwich structure with a dielectric layer sandwiched between two electrode layers, and a contoured through-hole drilled into the structure. Each layer is a few hundred micron thick and the hole diameter is also of approximately this size. The device operates at Ar flow rates of $\sim 1$ sccm with moderate electrode voltages $(\sim 1000 \mathrm{~V})$. Spectral measurements of the plume are used to determine its composition and calculate the electron excitation temperature. A two-dimensional computational model has been developed to provide a detailed description of plasma dynamics inside the MPT including power deposition, ionization, coupling of plasma phenomena with the high-speed flow, and propulsion system performance. Gas heating, primarily due to ion Joule heating, is found to have a strong influence on the overall discharge behavior.
\end{abstract}

\section{INTRODUCTION}

Small satellites (less than $100 \mathrm{~kg}$ mass) have recently gained interest for various commercial, military, and science space missions [1,2]. These satellites are power-limited: typically less than $1 \mathrm{~W} / \mathrm{kg}$ of spacecraft mass is available. Propulsion requirements can vary significantly, but are generally characterized by their very low thrust values $(\sim \mathrm{mN})$ and low impulse bits for attitude control $(\sim 10 \mu \mathrm{N} \cdot \mathrm{s})$. The scaling of traditional propulsion systems down in terms of power and size to suit the needs of small satellites is a major engineering challenge.

This is an Open Access article distributed under the terms of the Creative Commons Attribution-Noncommercial License 3.0, which permits unrestricted use, distribution, and reproduction in any noncommercial medium, provided the original work is properly cited. 
The extremely small dimensions of microdischarges combined with intense and controllable gas heating can be exploited in microthrusters technologies. Like conventional cold-gas thrusters, electrothermal microdischarge propulsion systems can have extremely low mass and volume footprints. The microdischarge operates in an abnormal glow mode with positive differential resistivity. An increase in input electrical power results in almost linear increase in the gas temperature; this property of microdischarges is a key feature that can be exploited in the MPT concept. The thrust from these devices is widely tunable by varying power levels.

\section{EXPERIMENTAL STUDIES}

Figure 1 shows the schematic of the MPT prototype used in the experimental studies. The MPT has a multilayer sandwich structure. It has three electrodes $e_{1}, e_{2}$, and $e_{3}$ which are $\sim 100 \mu \mathrm{m}$ thick and separated by $\sim 200 \mu \mathrm{m}$ thick dielectric layers. It has a narrow duct $\sim 100 \mu \mathrm{m}$ in diameter which expands to $\sim 330 \mu \mathrm{m}$ downstream of the throat. In terms of gas dynamics, the cavity inside the MPT resembles a converging-diverging (CD) nozzle that can accelerate the gas to supersonic velocities downstream of the throat. The mass of the assembled piece is $0.9 \mathrm{~g}$ and the total thickness is about $1.1 \mathrm{~mm}$.

The discharge is generated between $e_{1}$ and $e_{3}$. It heats the neutral gas which accelerates as it flows in the CD nozzle. The ion flow is also accelerated by the nozzle and the floating potential that exists between the MPT and the vacuum. The expulsion of hot gas and ions into the vacuum generates thrust. The MPT has potentially important advantages over competing concepts such as the microresistojet thruster [3] which also relies on preheating an expanding gas stream. The important difference between a resistojet and the MPT is the nature of power deposition into the gas.

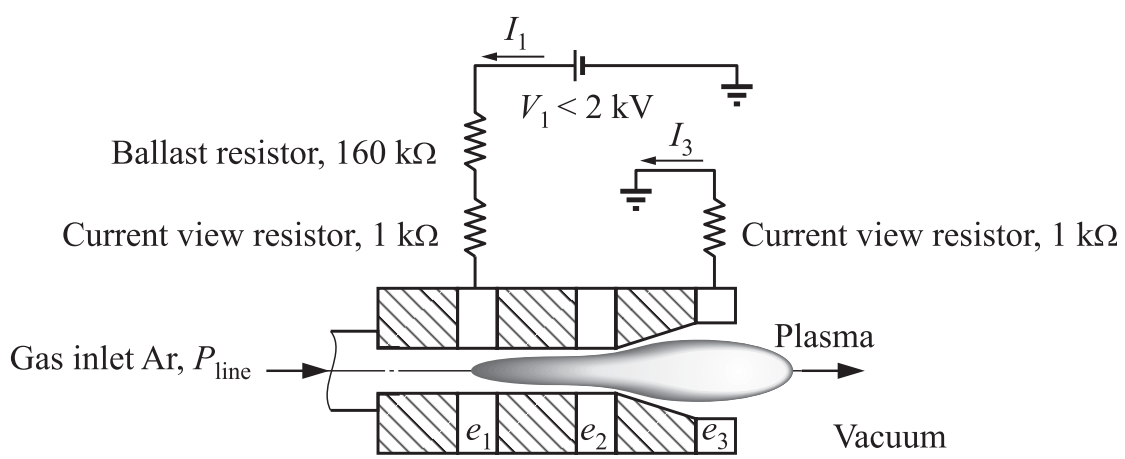

Figure 1 Schematic diagram of MPT with electrical circuit used in the experiments 

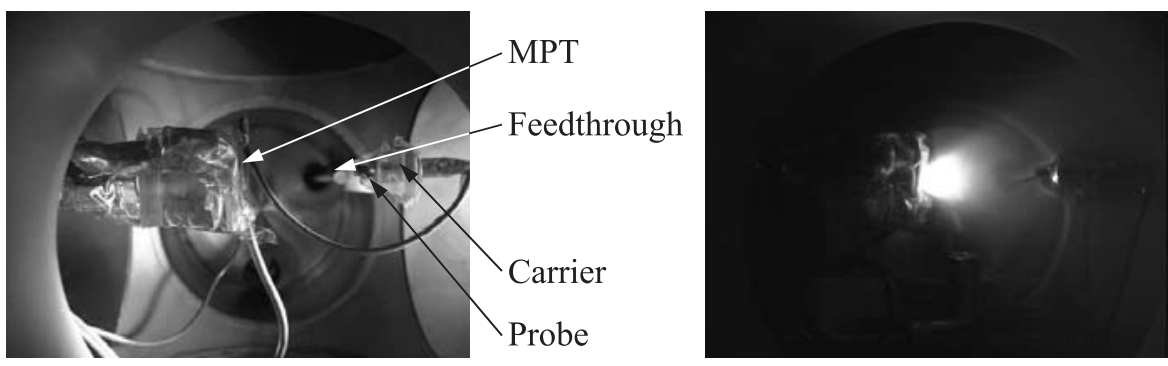

Figure 2 Microdischarge plasma thruster mounted inside the vacuum chamber with the ion probe $(a)$; and in operation $(b)$

In a resistojet, the heating element is essentially the hottest part of the system and heat addition to the gas occurs via conduction/convection to the gas stream. There is clearly an upper limit on the heating element temperature which, in turn, limits the amount of heat delivered to the gas.

In the MPT, the gas is heated directly by the plasma. Importantly, if the plasma thermal power deposition occurs far away from the surfaces, much higher thermal energies can be delivered to the gas. So far, the MPT has been operated using argon. When the argon flow rate is $5 \mathrm{sccm}$, the pressure in the cavity ranges from $\sim 100$ Torr upstream to $\sim 1$ Torr near the exit. Between $e_{2}$ and $e_{3}$, because the number density of gas species $(N)$ is low, the ratio of characteristic electric field strength $(E)$ to the number density of neutral species $E / N$ is high. This is favorable to enhance ionization processes in the discharge. Usually, at pressures as low as $\sim 1$ Torr and linear dimensions on the order of few hundred micron, the breakdown voltage is on the order of several kilovolts if the discharge is to be sustained only by secondary electron emissions at the cathode. The structure of the MPT helps overcoming such high voltage requirement to sustain the discharge. The high pressure $\left(\sim 100\right.$ Torr) between $e_{1}$ and $e_{2}$ helps reducing the breakdown voltage of gas inside the MPT. The region of the discharge between $e_{1}$ and $e_{2}$ can also supply electrons to the region between $e_{2}$ and $e_{3}$ to increase and sustain ionization processes in that region. In this way, the MPT can be stably operated using a noncorrosive/noncontaminating propellant at relatively low voltages.

To determine the ion distribution in the plume an ion probe was used. The probe consisted of a copper rod of radius $1 \mathrm{~mm}$ inserted into a glass tube that had $1 \mathrm{~mm}$ thick walls. The probe surface was biased at $-100 \mathrm{~V}$ and was mounted on a linear translation feedthrough, so that it could traverse normally to the plume axis in the horizontal plane. The probe was $4.4 \mathrm{~cm}$ away from the MPT exit when aligned with the plume axis. Figure $2 a$ shows a photograph of the experimental setup with the probe in place. Figure $2 b$ shows the MPT in operation. It is a 


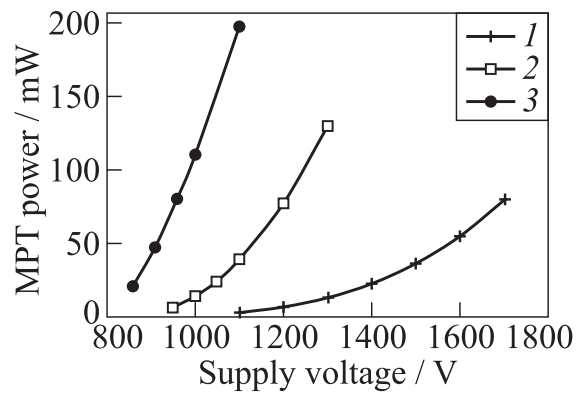

Figure 3 Variation of power with voltage at several flow rates through the MPT: $1-3 \mathrm{sccm} ; 2-4$; and $3-5 \mathrm{sccm}$

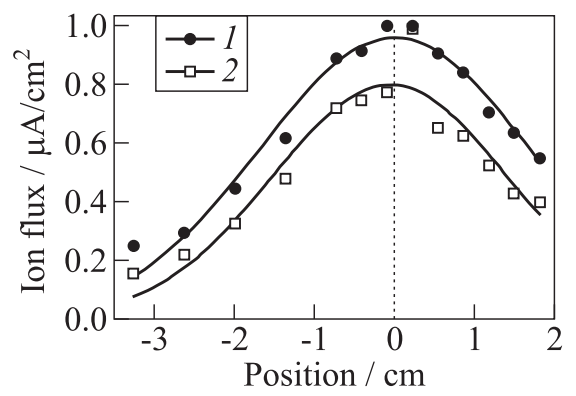

Figure 4 Ion current distribution in the plume: $1-250 \mathrm{~mW}$; and $2-180 \mathrm{~mW}$

highly underexpanded jet as the pressure at the exit is $\sim 1$ Torr and the pressure in the vacuum chamber is $\sim 10^{-3}$ Torr.

Figure 3 shows the MPT power depending on the supply voltage and flow rate. The typical operating power was between 50 and $500 \mathrm{~mW}$. The power is seen to increase with the flow rate at a fixed supply voltage. This implies that at higher flow rates, the increase in the number density of charge carriers outweighs the increase in collision frequency.

Figure 4 shows some results of the ion probe measurements. The total ion current in the plume was estimated from an analytic integral of a Gaussian fit to the probe data. For example, the total ion current at 250-milliwatt discharge power was $17.0 \mu \mathrm{A}$. Assuming that all the ions were $\mathrm{Ar}^{+}$, the ratio of ion fraction in the plume was $5.2 \cdot 10^{-5}$. Assuming that all the ions in the discharge were also $\mathrm{Ar}^{+}$, the ratio of ion current in the plume to discharge current was $5.6 \cdot 10^{-2}$. The low ion fraction implies that ion acceleration did not contribute significantly to the device thrust which comes mainly from gas dynamic expansion.

Emission spectroscopy was used to characterize the plume emerging from the MPT. The light from the plume was focused onto an optical fiber $(600 \mu \mathrm{m}$ in diameter) and transmitted to a 0.25 -meter spectrograph with an entrance slit width of $200 \mu \mathrm{m}$, and a grating with 1800 grooves $/ \mathrm{mm}$. The spectra were recorded by the spectrograph with a charge-coupled device (CCD) array detector; the exposure time ranged from $600 \mathrm{~ms}$ for the strongest lines up to $60 \mathrm{~s}$ for weaker lines. The spatial extent of the probe volume was determined by probing emission from an argon calibration lamp focused into a pinhole that had a diameter of $100 \mu \mathrm{m}$. The depth of field was found to be $3.03 \mathrm{~cm}$ and the imaged region along the plume axis was $260 \mu \mathrm{m}$. The plume spectra were recorded from the region $2 \mathrm{~mm}$ downstream of the exit plane. At this location, the length of 


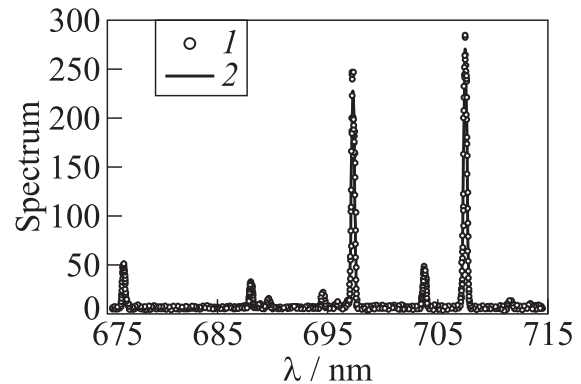

Figure 5 Experimental spectrum (1) and fit to the Doppler line shape (2)

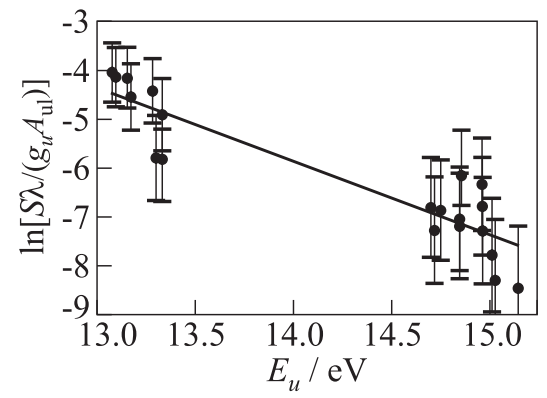

Figure 6 Boltzmann plot based on fitted line intensities: $T_{\text {fit }}=0.66 \mathrm{eV}$ $(7650 \mathrm{~K})$

the visible plume normal to its axis was estimated to be less than $3 \mathrm{~cm}$; so, the optical system collected light over the entire depth of the plume in these emission measurements.

The fixed pattern noise in the detector was subtracted and the detector and optical system response in the range 400-900 nm was calibrated with a black body source at $1473 \mathrm{~K}$. The spectral lines from $\mathrm{Ar}, \mathrm{Ar}^{+}$, and Mo (from electrode erosion) were observed. Figure 5 is a sample spectrum showing a total of 14 lines from neutral Ar with detectable intensity in this spectral range (not all the lines are apparent in the figure). The absolute positions of and spectroscopic data for the lines were taken from the NIST on-line atomic database [4]. The individual line intensities were determined by fitting Doppler profiles to the spectral data. The fitted line positions agree very well with the NIST data with a constant small offset of $0.827 \mathrm{~nm}$ (arising from a small offset in the spectrometer wavelength calibration) providing confidence that the lines were assigned correctly.

Using data on neutral Ar lines in $\pm 20 \mathrm{~nm}$ wavelength intervals around 710 and $811 \mathrm{~nm}$, a Boltzmann plot to determine the excitation temperature of $\mathrm{Ar}$ electronic states was constructed. The results of this analysis are shown in Fig. 6 . The error bars correspond to $\pm 15 \%$ and are principally due to the estimated uncertainty of the Einstein coefficients $A$ for these transitions. The NIST online data base quotes the absolute accuracy of the Einstein coefficients $A$ for the observed transitions as $\mathrm{C}(< \pm 25 \%)$ or $\mathrm{D}+(< \pm 40 \%)$ [4]. The present estimate of $\pm 15 \%$ error is based on the assumption that the relative precision of the data is somewhat better than the absolute accuracy. The measurements are consistent with an "excitation" temperature of approximately $0.66 \mathrm{eV}(7650 \mathrm{~K})$. This is very different from the gas kinetic temperature which is expected to be $\sim 1000 \mathrm{~K}$. Note that the gas temperature could not be determined from the recorded spectral line shape as the measured line shape was determined largely by the spectrometer 
resolution. The excitation temperature of these electronic states in the plume was not expected to be representative of the electron temperature in the discharge inside the MPT which was probably several electronvolts. It is possible that the result represents the free electron temperature in the weakly ionized plume, but the measurements must be interpreted with caution as they represent emission from high lying excited states of neutral Ar. As seen in Fig. 6, the emitting states are $13-15 \mathrm{eV}$ above the ground state. Hence, electron impact ionization of these states would be from electrons at the high-energy tail of the energy distribution which is likely to be depleted and out of equilibrium with the remainder of the distribution.

\section{$3 \quad$ NUMERICAL STUDIES}

In the MPT, the microdischarge plasma is in a highly nonequilibrium state with disparate electron and gas temperatures and nonequilibrium finite-rate chemical effects. The gas flow is characterized by low-Reynolds number, viscosity dominated effects combined with high-Mach-number compressibility effects owing to the expansion of the relatively high-pressure ( $\sim 100$ Torr $)$ micronozzle gas stream into vacuum. Both plasma and flow phenomena are strongly coupled: the plasma causes gas heating which modifies the gas density and, hence, the flow field; the gas flow velocities, in turn, affect the distributions of species and temperature in the discharge plasma. The integrated plasma-flow model used in this study was described in detail in [5].

A pure argon plasma-gas chemistry $[6,7]$ was used which comprised six species: electrons $(e)$, atomic argon ions $\left(\mathrm{Ar}^{+}\right)$, molecular argon ions $\left(\mathrm{Ar}_{2}^{+}\right)$, electronically excited atoms $\left(\mathrm{Ar}^{*}\right)$, electronically excited molecules $\left(\mathrm{Ar}_{2}^{*}\right)$, and the background argon atoms (Ar). Dimer species were included because of the relatively high pressures in the upstream section of the micronozzle ( $\sim 100$ Torr $)$. At solid surfaces, all excited species and charged species were assumed to get quenched with unity sticking coefficient. Upon quenching at surfaces, each dimer ion and excited species was assumed to return to plasma as a pair of ground state neutral Ar atoms, while the monomer species returned as single Ar atoms.

Conservation equations for the gas mass density, mass-averaged gas velocity, and the gas energy were solved using compressible Navier-Stokes equations in axisymmetric form. On the one hand, the flow solution influenced the plasma discharge through the pressure, temperature, and velocity fields appearing in the plasma governing equations. On the other hand, electrostatic forces and electrothermal heating acted as external body forces and external heat source, respectively, in the Navier-Stokes equations. The Joule heating terms appearing in the electron energy equation were evaluated using a species flux reconstruction approach [8]. 


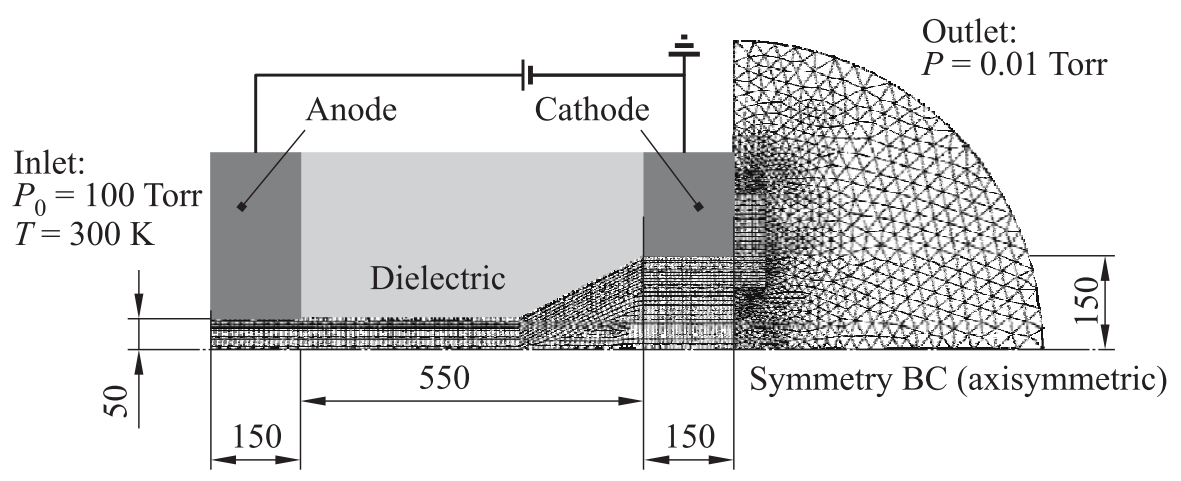

Figure 7 Schematic of the MPT device and computational mesh. The geometry is cylindrically symmetric. Dimensions are in microns

Both plasma governing equation and compressible flow Navier-Stokes equations were spatially discretized using a cell-centered finite volume approach on an unstructured mesh with mixed mesh cell types. A steady-state solution was sought in all cases. Both plasma and flow governing equations were solved as transient problems with time-stepping of the solution to a steady state. The plasma governing equations were solved using an implicit time-discretization approach with local linearization of the governing equations at each time step. For the flow governing equations, the inviscid flux terms were evaluated with the Advection Upstream Splitting Methods (AUSM) [9] and the viscous flux terms were evaluated using the Haselbacher approach [10]. The flow equations were also solved using implicit time discretization, with a dual-time stepping approach to iteratively solve for the solution at each time step. The viscous terms in the flow equations required computation of the solution variable gradients at cell centers. To reconstruct these gradients based on cell-centered values of the corresponding variables, a Green-Gauss method was used. Finally, the compressible flow could develop shock discontinuities. Therefore, the Venkatakrishnan flux limiter approach [11] was used to stabilize and produce monotone solutions in the presence of such discontinuities.

Figure 7 shows the geometry of the MPT used in the simulations. The geometry consisted of an axisymmetric constant area "pipe" section of 500micron length, followed by a diverging section $200 \mu \mathrm{m}$ long which was terminated by a 150 -micron long constant area section. The radius of the upstream constant area pipe section was $50 \mu \mathrm{m}$, and the exit section was $150 \mu \mathrm{m}$ in radius. The ring-shaped electrodes had an axial thickness of $150 \mu \mathrm{m}$, while the dielectric layer had an axial thickness of $550 \mu \mathrm{m}$. The mesh consisted of about 3600 cells which included a combination of triangles and quadrilaterals. 
The flow direction in Fig. 7 was from left to right. The flow entered the constant-area tube section on the left and exited the domain along the curved (arc-shaped) boundary on the right. Four boundary sections were not modeled as solid walls for the gas discharge governing equations. Zero-flux boundary conditions were imposed at the boundary section formed by the symmetry axis. For numerical stability reasons, zero-flux boundary conditions were also used for the gas discharge governing equations at the inlet and outer cathode surface boundaries. These boundaries were sufficiently away from the main discharge region and did not influence the results. The far-field boundary on the right side of the computational domain was modeled as an "outflow" boundary, where the plasma variables (species number densities and electron energy density) were convected away by the gas flow, while zero-flux boundary conditions were imposed for the Poisson equation. The value of the plasma variable from the adjacent interior cell was used to interpolate the flux at this far-field boundary. The power input was provided by applying a fixed positive direct-current (DC) voltage at the anode (without ballast resistance), while the cathode was grounded. For all the calculations shown here, the secondary electron emission coefficient was set at a value of 0.03 , corresponding to nickel surface interacting with argon plasma [12].

The inlet total (stagnation) pressure was 100 Torr (for the base case), and a small but nonzero outlet pressure (0.05 Torr) was required to stabilize the numerical scheme in the "vacuum" part of the domain. The inlet gas static temperature was fixed at $300 \mathrm{~K}$. The solid wall temperature was fixed at $300 \mathrm{~K}$. The inlet flow velocity was computed self-consistently using the inlet conditions, the inlet static pressure extrapolated from the interior of the domain, and isentropic expansion relations. No-slip boundary conditions were applied at solid walls for the momentum equation. The Knudsen numbers for the flow were sufficiently low over much of the MPT device length (about 0.01 at the inlet to about 0.08 at the exit plane) that slip-flow and wall-temperature jump boundary conditions were not necessary. This has been verified by running simulations with the appropriate jump boundary conditions.

Table 1 lists the discharge conditions for which the results are presented here. The effect of other parameters was also studied and more detailed results are given in [5]. For the base case, the discharge voltage was set to $750 \mathrm{~V}$, and the computed discharge current was $0.87 \mathrm{~mA}$. The stagnation pressure (100 Torr) and static temperature $(300 \mathrm{~K})$ imposed at the inlet determined the inlet velocity which was computed at about $100 \mathrm{~m} / \mathrm{s}$ corresponding to a flow rate of $5.2 \mathrm{sccm}$. Representative plasma properties at steady state for this case are shown as false color images in Fig. 8. The space charge density is high enough that the bulk plasma extends well into the diverging part of the micronozzle creating a hollow-cathodelike annular cathode sheath. Under these operating conditions, the cathode sheath was about $100 \mu \mathrm{m}$ thick (see electrostatic potential in Fig. 8a), occupying a significant fraction of the total discharge volume. In 
Table 1 Operating conditions considered for the results presented in this work. The characteristic discharge dimension $D$ is taken to be the thickness of the dielectric layer located between the electrodes $(550 \mu \mathrm{m})$ and the characteristic pressure is taken to be the inlet total pressure

\begin{tabular}{|c|c|c|c|c|c|c|c|c|}
\hline \multirow[b]{2}{*}{ Case } & \multirow{2}{*}{$\begin{array}{c}\text { Inlet total } \\
\text { pressure, } \\
\text { Torr }\end{array}$} & \multirow{2}{*}{$\begin{array}{c}\text { PD, } \\
\text { Torr } \cdot \mathrm{cm}\end{array}$} & \multirow{2}{*}{$\begin{array}{l}\text { Flow } \\
\text { rate, } \\
\text { sccm }\end{array}$} & \multirow{2}{*}{$\begin{array}{c}\text { Voltage, } \\
\text { V }\end{array}$} & \multirow{2}{*}{$\begin{array}{c}\text { Current, } \\
\mathrm{mA}\end{array}$} & \multirow{2}{*}{$\begin{array}{c}\text { Power, } \\
\text { mW }\end{array}$} & \multicolumn{2}{|c|}{ Computed } \\
\hline & & & & & & & $\begin{array}{c}\text { thrust, } \\
\mu \mathrm{N}\end{array}$ & $\begin{array}{c}\text { power, } \\
\mathrm{mW}\end{array}$ \\
\hline Base case & 100 & 5.5 & 5.2 & 750 & 0.87 & 650 & 100 & 650 \\
\hline $\begin{array}{l}\text { Larger power } \\
\text { input }\end{array}$ & 100 & 5.5 & 5.2 & 1000 & 1.8 & 1800 & 128 & 1800 \\
\hline $\begin{array}{l}\text { Higher cathode } \\
\text { temperature }\end{array}$ & 100 & 5.5 & 5.2 & 750 & 0.72 & 540 & 112 & 540 \\
\hline
\end{tabular}

the cathode fall, the potential dropped by nearly $750 \mathrm{~V}$ over $\sim 100 \mu \mathrm{m}$ producing a characteristic field strength of $\sim 75 \mathrm{kV} / \mathrm{cm}$ and a reduced electric field $(E / N)$ of $\sim 10^{5} \mathrm{Td}$ (Townsend). Electron density contours are presented in Fig. $8 b$ and show two local maxima. The first peak is inside the constant area tube section $\left(\sim 7 \cdot 10^{19} \mathrm{~m}^{-3}\right)$, and the second higher peak is in the diverging section of the nozzle $\left(\sim 3 \cdot 10^{20} \mathrm{~m}^{-3}\right)$. The well-defined cathode sheath structure observed in Fig. $8 a$ is apparent in the density contours with an abrupt drop in the electron densities in the diverging micronozzle section. The MPT discharge clearly operates in the glow discharge mode rather than the Townsend/predischarge mode. The electron temperatures are displayed in Fig. $8 c$. The electron temperature is only shown in regions of the discharge where the electron number density is greater than $3 \cdot 10^{17} \mathrm{~m}^{-3}$, i. e., $10^{-3}$ of the peak value of the electron number density. The electron energy content is negligible in the rest of the domain. The electron temperature remains nearly uniform $(\sim 2 \mathrm{eV})$ in the bulk plasma over most of the constant area tube section, and gradually increases in the diverging section of the device to temperatures around $3 \mathrm{eV}$ at the cathode sheath edge.

The electron generation rate in the discharge through gas-phase reactions is shown in Fig. 8d. Significant generation of electrons is observed over the entire micronozzle with maximum generation observed in the diverging section. This location corresponds to a relatively higher electron temperature $(\sim 3 \mathrm{eV})$ and reasonably high background densities in the expanding gas flow. The argon monomer ion $\left(\mathrm{Ar}^{+}\right)$and dimer ion $\left(\mathrm{Ar}_{2}^{+}\right)$number densities are shown in Figs. $8 e$ and $8 f$, respectively. Atomic argon ions constitute the dominant ion species in the microdischarge. High background densities favor three-body reactions that form dimer species. Therefore, most dimer ions are located in the constant area tube section where the pressure is relatively high. The dielec- 
Electrostatic potential / V

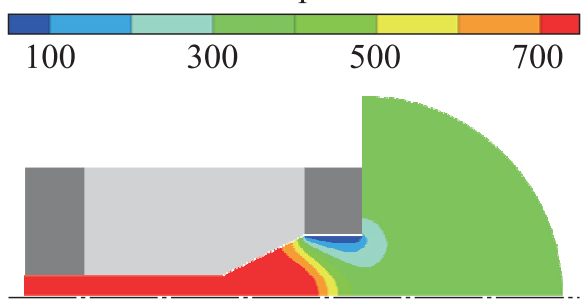

(a)

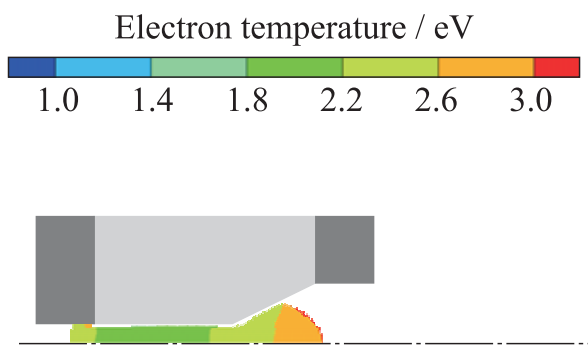

(c)

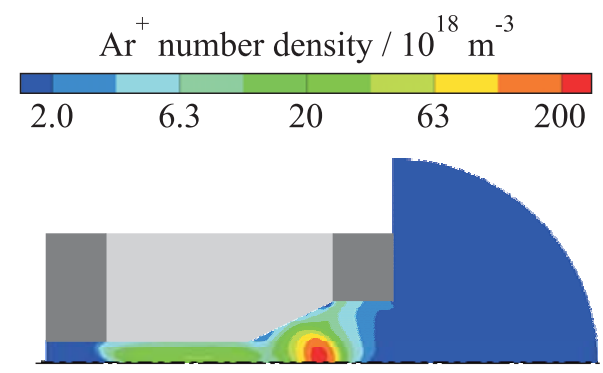

$(e)$
Electron number density $/ 10^{18} \mathrm{~m}^{-3}$

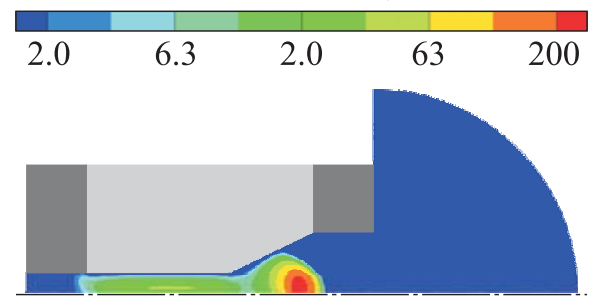

(b)

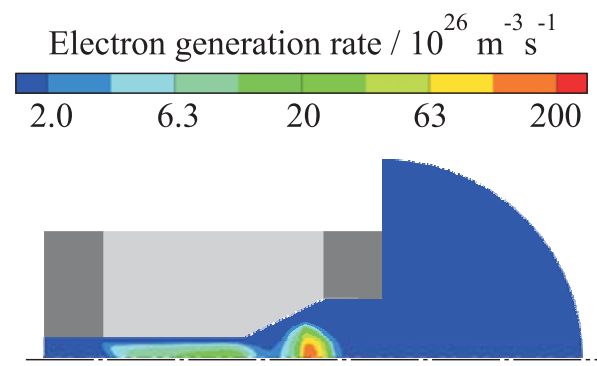

(d)

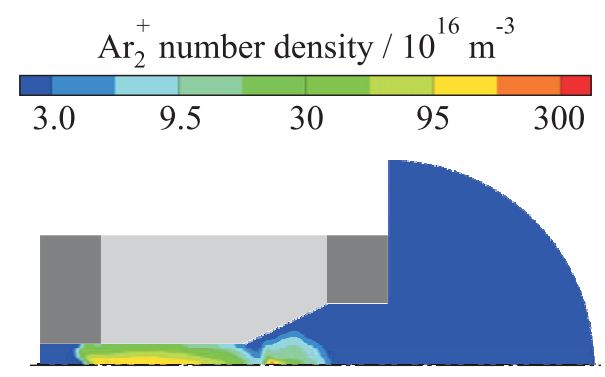

$(f)$

Figure 8 Plasma properties in the MPT: $(a)$ electrostatic potential; $(b)$ electron number density; $(c)$ electron temperature; $(d)$ electron generation rate; $(e)$ Ar number density; and $(f) \mathrm{Ar}_{2}$ number density. The inlet total pressure is 100 Torr $(13.3 \mathrm{kPa})$ and the mass flow rate is $5.2 \mathrm{sccm}$. The applied potential difference between the electrodes is $750 \mathrm{~V}$ and the power input is $650 \mathrm{~mW}$. (Refer $\mathrm{KC}$ et al., p. 594.) 
tric surfaces support a net negative charge owing to electron trapping which, in turn, supports a positive sheath over the entire dielectric length between the anode and the cathode. This is apparent from the excess of positive ions compared to electrons, just above the dielectric surfaces. The net negative charge
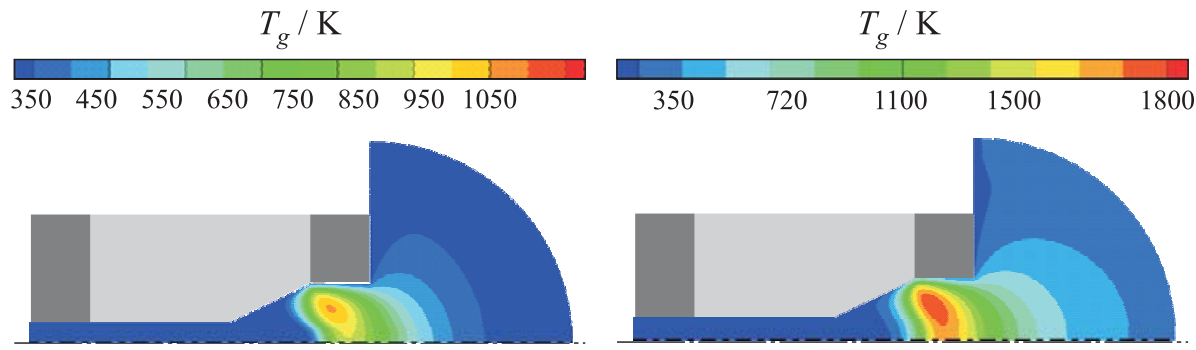

(a)

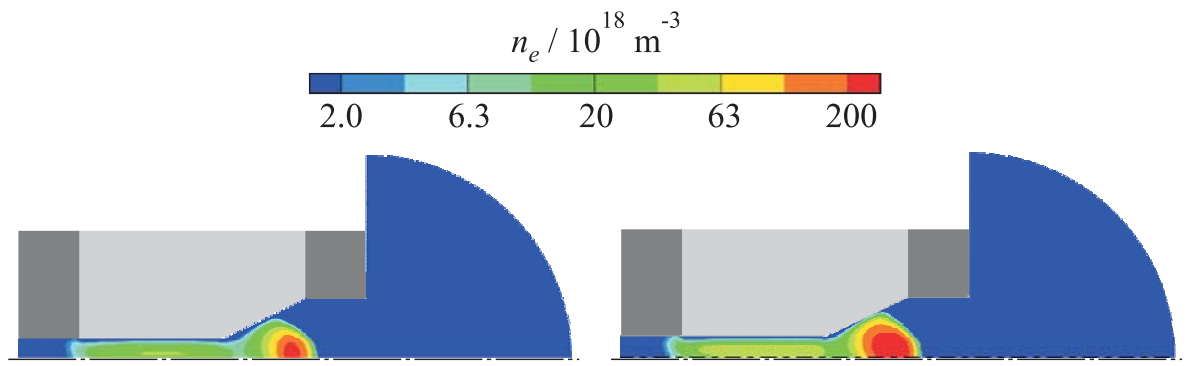

(b)

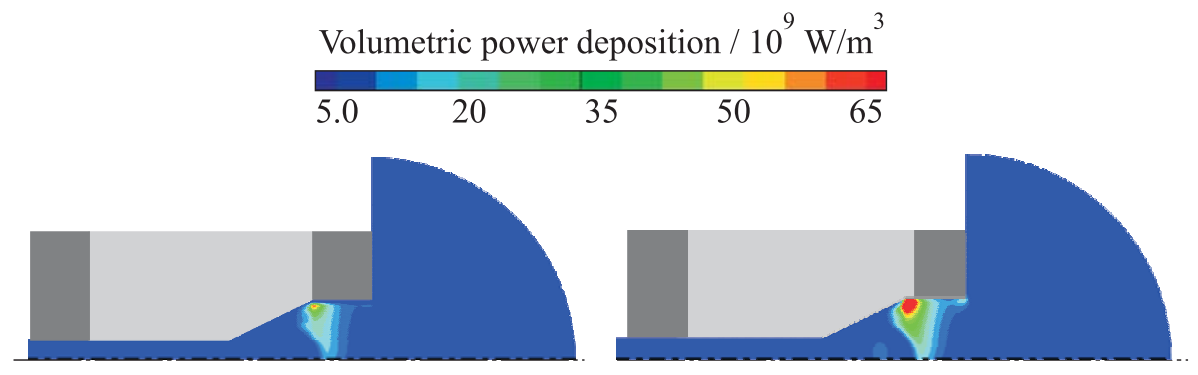

(c)

Figure 9 Effect of applied voltage and discharge power on $(a)$ gas temperature; (b) electron density; and $(c)$ volumetric power deposition in the MPT for two cases: left column $-V_{\text {applied }}=750 \mathrm{~V}$, power $=650 \mathrm{~mW}$; and right column $-V_{\text {applied }}=1000 \mathrm{~V}$, power $=1800 \mathrm{~mW}$. (Refer KC et al., p. 595.) 
at the dielectric enforces the electron wall flux to equal the total positive ion wall flux (at steady state); so, no net current is drawn through the dielectric surfaces.

Figure 9 shows the effects of power input on discharge characteristics and flow properties. The results are presented for the base case, where the discharge voltage is fixed at $750 \mathrm{~V}$ and the discharge power is $650 \mathrm{~mW}$, and for the case where the discharge voltage is fixed at $1000 \mathrm{~V}$ with a corresponding power of $1800 \mathrm{~mW}$. In both cases, the inlet total pressure was 100 Torr and the flow rate was $5.2 \mathrm{sccm}$.

Gas temperatures are compared in Fig. $9 a$. The peak temperature for the high power case is $1850 \mathrm{~K}$ compared to $950 \mathrm{~K}$ for the base case. Figure $9 b$ shows that the plasma is more intense for higher power inputs, i.e., the ionized species number densities are higher. Electron number densities increase by a factor of $\sim 2$ as the power input increases from 650 to $1800 \mathrm{~mW}$. Contours of the power deposition in the MPT are shown in Fig. $9 c$. The net power deposition into neutral gas scales almost linearly with the power input. Importantly, these results show that changing the external power input is an effective method to control the level of gas heating in the discharge which, in turn, affects the gas temperature and the thrust produced by the device. This is a key feature of the present MPT concept - the computed thrust increases to $128 \mu \mathrm{N}$ for the high power case which corresponds to a specific impulse of $95 \mathrm{~s}$.

In practice, a large fraction of the input power is lost to the walls; so, the cathode temperature is expected to be significantly higher than the constant temperature of $300 \mathrm{~K}$ assumed in the above computations. An accurate estimate of the cathode temperature would require solving an energy balance equation for the entire MPT device (including solid materials). The effect of the cathode temperature on the MPT discharge parameters and performance was studied by comparing the base case solution to a case where the cathode temperature was fixed at $1000 \mathrm{~K}$. All other conditions were kept the same as in the base case. Figure $10 a$ shows the gas temperature contours for the two cases. As might be expected, the spatial distribution of computed gas temperature is strongly dependent of the assumed cathode temperature. The peak gas temperature reaches $1150 \mathrm{~K}$ with the cathode temperature fixed at $1000 \mathrm{~K}$, compared to $950 \mathrm{~K}$ for the base case, and the region of high gas temperatures occupies a larger fraction of the MPT volume than for the base case. Increasing the cathode temperature reduces the temperature gradients at the cathode surface, thereby decreasing the net thermal loss.

The axial velocity contours are shown for the two cases in Fig. 10b. As the cathode temperature is increased, the reduced heat losses allow for an increased expansion of the gas in the diverging section of the micronozzle. The computed thrust, therefore, increases from $100 \mu \mathrm{N}$ for the base case to $112 \mu \mathrm{N}$ when the cathode temperature is $1000 \mathrm{~K}$. It should be noted that the power input has 


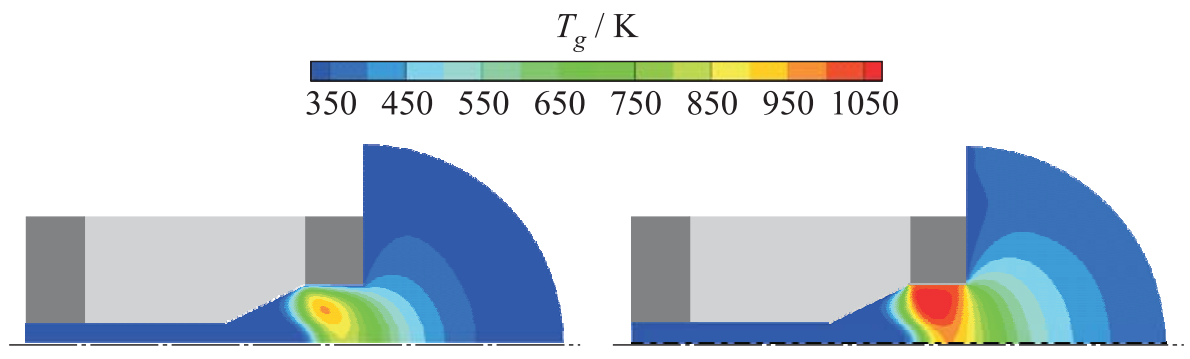

(a)

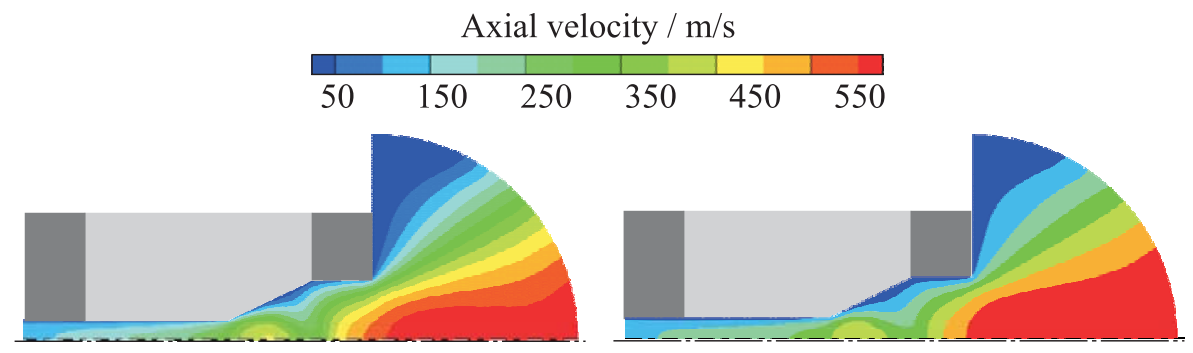

(b)

Figure 10 Effect of cathode temperature on $(a)$ gas temperature and $(b)$ axial velocity for two cases: left column $-T_{\text {cathode }}=300 \mathrm{~K}, P_{\text {input }}=650 \mathrm{~mW}$, and thrust $=100 \mu \mathrm{N}$; and right column $-T_{\text {cathode }}=1000 \mathrm{~K}, P_{\text {input }}=540 \mathrm{~mW}$, and thrust $=112 \mu \mathrm{N}$. (Refer $\mathrm{KC}$ et al., p. 597.)

decreased to a value of $540 \mathrm{~mW}$ at the higher cathode temperature, compared to $650 \mathrm{~mW}$ for the base case. The higher gas temperatures (and, therefore, lower background Ar densities) result in a decrease in ionization. The lower power/current can be attributed to the lower charge densities and, hence, to the lower plasma conductivity.

\section{CONCLUDING REMARKS}

The MPT is an electrothermal micropropulsion device that was designed and studied with the goals of minimizing its size, mass, operating power, and propellant consumption. The MPT was successfully operated in the lab at low flow rates of $\sim 1 \mathrm{sccm}$. The most stable operation was obtained with the upstream electrode $\left(e_{1}\right)$ as the anode, the downstream electrode $\left(e_{3}\right)$ as the cathode, and the middle electrode $e_{2}$ left floating. The MPT operates as an electrothermal 
thruster because most of the momentum thrust of the MPT is generated by the expulsion of hot neutral argon atoms. Measurements with the ion probe revealed that there was also a small fraction of ions in the plume. Hence, the MPT is able to expel ions without using an external magnetic field or an external electron emitter. The comparison of current voltage characteristics of the MPT (not presented here) with ion probe measurements shows that the net ion current in the plume is also considerably less than the discharge current. The presence of ions in the plume was confirmed by emission spectroscopy. Molybdenum atoms in the plume detected from their spectra implies that the plume eroded the exposed regions of the electrodes. Using the emission spectra, the electronic excitation temperature of neutral Ar was determined to be $\sim 0.7 \mathrm{eV}$ $(\sim 7700 \mathrm{~K})$. The thrust from the MPT is yet to be measured experimentally. The material selection for the electrodes also needs to be addressed in the future.

A detailed computational model was also used to study microdisharge and flow interaction phenomena for micropropulsion applications. The model consisted of a plasma module coupled to a flow module and was solved on a hybrid unstructured mesh framework. The plasma module provided a self-consistent, multispecies, multitemperature description of the microdischarge phenomena while the flow module provided a description of the low-Reynolds-number compressible flow through the micropropulsion system.

The coupled plasma-flow simulations showed that the electrostatic component of thrust was negligible for the current MPT configuration. Electrothermal heating was due primarily to the ion Joule heating occurring near the cathode surfaces. A large part of the input power was, therefore, deposited into the walls rather than going to neutral gas heating. Future work must explore strategies that will allow for power deposition away from the wall, near the centerline. For example, radio-frequency operation of the microdischarge may help reducing the thermal load on the microdischarge and increasing the direct power deposition into the neutral gas.

For a discharge voltage of $750 \mathrm{~V}$, a power input of $650 \mathrm{~mW}$, and an argon flow rate of $5.2 \mathrm{sccm}$, the computed specific impulse of the device was $74 \mathrm{~s}$, i. e., a factor of $\sim 1.5$ higher than that for the cold-gas micronozzle. For these conditions, charged species densities on the order of $5 \cdot 10^{20} \mathrm{~m}^{-3}$ and peak gas temperatures of $\sim 1000 \mathrm{~K}$ were predicted. The microdischarge remained mostly confined inside the micronozzle and operated in an abnormal regime. Additional simulation studies on the MPT indicated that the power input had a strong influence on overall discharge properties. The net power deposition into the neutral gas scaled with the power input, providing a method of controlling the gas temperature and the thrust level of the MPT. This feature constitutes an important advantage over traditional cold-gas thrusters.

The sensitivity of the results described above to important uncertain parameters of the model has been also studied. A higher cathode temperature was 
found to reduce the heat losses at solid walls and was beneficial for the thruster performance, though there was obviously an upper limit to avoid damage to the cathode. The cathode temperature could be determined self-consistently by solving the solid thermal conduction equation in the dielectric and electrode material to quantify the overall thermal field in the MPT device. The thermal loads from the plasma at the wall surfaces will drive heat transfer into the device structure potentially resulting in "hot spots" that can be investigated through the simulations. These coupled multiphysics simulation results would, in turn, provide an informative approach to the better design of the MPT.

\section{ACKNOWLEDGMENTS}

This work was supported by AFOSR under grant FA9550-06-1-0176.

\section{REFERENCES}

1. Micci, M. M., and A.D. Ketsdever. 2000. Micropropulsion for small spacecraft. Progress in astronautics and aeronautics ser. Reston, VA: AIAA.

2. Sweeting, M. N. 2000. Space at surrey: Micro-mini-satellites for affordable access to space. Air Space Europe 2:38-52.

3. Coxhill, I. G., and D. Gibbon. 2005. A xenon resistojet propulsion system for microsatellites. AIAA Paper No. 2005-4260.

4. Ralchenko, Yu., A.E. Kramida, J. Reader, and NIST ASD Team. 2008. NIST Atomic Spectra Database (version 3.1.5) [Online]. Available: http:// physics.nist.gov/asd3 [2009, May 6]. Gaithersburg, MD: National Institute of Standards and Technology.

5. Deconinck, T. 2008. Simulation studies of direct-current microdischarges for electric propulsion. Ph.D. Thesis. University of Texas at Austin.

6. Lay, B., R.S. Moss, S. Rauf, and M. J. Kushner. 2003. Breakdown processes in metal halide lamps. Plasma Sources Sci. Technol. 12:8-21.

7. Hagelaar, G. J. M., and L. C. Pitchford. 2005. Solving the Boltzmann equation to obtain electron transport coefficients and rate coefficients for fluid models. Plasma Sources Sci. Technol. 14:722-33.

8. Deconinck, T., S. Mahadevan, and L.L. Raja. 2009. Discretization of the Joule heating term in plasma discharge fluid models in unstructured meshes. J. Comput. Phys. 228(12):4435-43.

9. Liou, M.-S., and C. J. Steffen. 1993. A new flux-splitting scheme. J. Comput. Phys. 107:23-39. 
10. Haselbacher, A., and J. Blazek. 2000. Accurate and efficient discretizations of Navier-Stokes equations on mixed grids. AIAA J. 38:2094-102.

11. Venkatakrishnan, V. 1995. Convergence to steady state solutions of the Euler equations on unstructured grids with limiters. J. Comput. Phys. 118:120-30.

12. Lieberman, M. A., and A. J. Lichtenberg. 1994. Principles of plasma discharges and materials processing. New York: John Wiley \& Sons. 